\title{
Modified variational iteration and homotopy analysis method for solving variable coefficient variant boussinesq system
}

\author{
Fadhil H. Easif a, Saad A. Manaa ${ }^{b}$, and Ahmed J. Sabali ${ }^{*}$ \\ $a, b, c$ Faculty of Science, University of Zakho, Zakho, Kurdistan Region, Iraq \\ Emails: a fadhil.easif@uoz.edu.krd, b saad.manaa@uoz.edu.krd , c ahmed.sabali@uoz.edu.krd
}

\begin{abstract}
In this paper, Modified Variational Iteration Method (MVIM) and Homotopy Analysis Method (HAM) are used to find approximate solutions for the Variable-Coefficient Variant Boussinesq System the (VCVB) system is able to describe the nonlinear and dispersive long gravity waves in shallow water traveling in two horizontal directions with varying depth, as an example we took the Boussinesq-Burgers (B-B) system, (B-B) system arise in the study of fluid flow and describing the long-wave propagation of shallow water waves. The solutions of these equations helpful for the coastal and civil engineering's
\end{abstract}

Keywords: Variational Iteration, Homotopy Analysis, Variable-Coefficient Variant Boussinesq System, BoussinesqBurgers $(B-B)$.

2010 MSC: 65C20, 93C10, 33F05, 34K2

\section{Introduction}

Most of the phenomena that arise in mathematical physics and engineering fields can be modelled by ordinary or partial differential equations. They are ubiquitous in science and engineering, as well as economics, social science, biology, business, health care, etc., which most of them are nonlinear differential equations [1]. Also, we have the mathematical representations of many physical systems [2,3] It is not easy to obtain closed form solutions for such problems. In most cases, only approximate solutions either analytical ones or numerical ones can be obtained. For that reason, the nonlinear equations should be solved using special methods.

In this paper, the MVIM and HAM are applied to, the Variable-Coefficient Variant Boussinesq System [4],

$$
\begin{gathered}
u_{t}+\alpha_{1}(t) v_{x}+\beta_{1}(t) u u_{x}+\gamma_{1}(t) u_{x x}=0 \\
v_{t}+\alpha_{2}(t) u v_{x}+\beta_{2}(t) v u_{x}+\gamma_{2}(t) v_{x x}+p(t) u_{x x x}=0
\end{gathered}
$$

where $\alpha_{i}(t), \beta_{i}(t), \gamma_{i}(t), i=1,2$ and $p(t)$ are arbitrary functions of $t$ and all of them are smooth functions (analytic functions) of the time t with $\gamma_{1}(t), \gamma_{2}(t)$ and $\mathrm{p}(\mathrm{t})$ representing different diffusion strengths. Where $\boldsymbol{x}$ and $\boldsymbol{t}$ represent the normalized space and time respectively, $\boldsymbol{u}(\boldsymbol{x}, \boldsymbol{t})$ is the horizontal velocity field (at the leading order it is the depth-averaged horizontal field) and $\boldsymbol{v}(\boldsymbol{x}, \boldsymbol{t})$ denotes the height of the water surface above a horizontal bottom.

\footnotetext{
${ }^{*}$ Corresponding author

Email address: ahmed.sabali@uoz.edu.krd (Ahmed J. Sabali)

doi:10.31559/glm2020.8.1.4

Received: 17 Nov 2019: Accepted 19 Feb 2020
} 


\section{Basic Idea}

\subsection{Modified Variational Iteration Method (MVIM)}

Consider the system in an operator form

$$
\begin{aligned}
& L_{t} u+L_{x} v+N_{1}(u, v)=g_{1}, \\
& L_{t} v+L_{x} u+N_{2}(u, v)=g_{2},
\end{aligned}
$$

With the initial conditions:

$$
u(x, 0)=f_{1}(x), \quad v(x, 0)=f_{2}(x) .
$$

Without loss of generality, $L_{t}$ and $L_{x}$ are considered to be first order partial differential operators, $N_{1}$ and $N_{2}$ are nonlinear operators, and $g_{1}$ and $g_{2}$ are source terms.

Using the same procedure of MVIM, as in [5]. Two recursive relations can be constructed as follows:

$$
U_{k+1}=U_{k}-\int_{0}^{t}\left\{L_{x}\left(V_{k}-V_{k-1}\right)+\left(G_{k}-G_{k-1}\right)\right\} d \tau \quad k \geq 0,
$$

and

$$
V_{k+1}=V_{k}-\int_{0}^{t}\left\{L_{x}\left(U_{k}-U_{k-1}\right)+\left(H_{k}-H_{k-1}\right)\right\} d \tau \quad k \geq 0,
$$

Where

$$
U_{-1}=0, U_{0}=g_{1}, U_{1}=U_{0}-\int_{0}^{t}\left\{L_{x}\left(V_{0}-V_{-1}\right)+\left(G_{0}-G_{-1}\right)-g_{1}\right\} d \tau,
$$

and $G_{k}(x, t)$ is obtained from

$$
N_{1} U_{k}(x, t)=G_{k}(x, t)+O\left(t^{k+1}\right) .
$$

and

and $H_{k}(x, t)$ is obtained from

$$
V_{-1}=0, V_{0}=g_{2}, V_{1}=V_{0}-\int_{0}^{t}\left\{L_{x}\left(U_{0}-U_{-1}\right)+\left(H_{0}-H_{-1}\right)-g_{2}\right\} d \tau,
$$

$$
N_{2} U_{k}(x, t)=H_{k}(x, t)+O\left(t^{k+1}\right)
$$

Equation $(4,5)$ can be solved iteratively to obtain an approximate solution that takes the form

$$
\begin{aligned}
& u(x, t) \cong U_{k}(x, t), \\
& v(x, t) \cong V_{k}(x, t),
\end{aligned}
$$

where $k$ is the final iteration step

Also, the advantages of MVIM are summarized in four points as in [5].

\subsection{Homotopy Analysis Method (HAM)}

We will examine the system of nonlinear partial differential equations by using Homotopy analysis method as in $[6,7]$, The general form of system of the partial differential equation is

$$
\begin{aligned}
& N_{1}[u(x, t, v)]+S_{1}(x, t)=0 \\
& N_{2}[v(x, t, u)]+S_{2}(x, t)=0 ; \quad x \in \Omega
\end{aligned}
$$

where $N_{1}, N_{2}$ are nonlinear operators, $u(x, t, v), v(x, t, u)$ are unknown functions and $S_{1}(x, t), S_{2}(x, t)$ are known functions, and $x, t$ are the spatial and temporal respectively. Using the same procedure [6,7], we can conclude two formulas:

$$
u_{m}(x, t)=\chi_{m} u_{m-1}(x, t)+c_{1} L^{-1}\left[R_{1, m}\left(\vec{u}_{m-1}, \vec{v}_{m-1}\right)\right], m \geq 1,
$$




$$
v_{m}(x, t)=\chi_{m} v_{m-1}(x, t)+c_{2} L^{-1}\left[R_{2, m}\left(\vec{u}_{m-1}, \vec{v}_{m-1}\right)\right], m \geq 1,
$$

where

$$
\begin{aligned}
\chi_{m} & = \begin{cases}0, & m \leq 1, \\
1, & m>1 .\end{cases} \\
R_{1, m}\left(\vec{u}_{m-1}, \vec{v}_{m-1}\right) & =\left.\frac{1}{(m-1) !} \frac{\partial^{m-1} N_{1}[\phi(x, t ; q)]}{\partial q^{m-1}}\right|_{q=0},
\end{aligned}
$$$$
R_{2, m}\left(\vec{u}_{m-1}, \vec{v}_{m-1}\right)=\left.\frac{1}{(m-1) !} \frac{\partial^{m-1} N_{2}[\psi(x, t ; q)]}{\partial q^{m-1}}\right|_{q=0} .
$$

Then, computing the approximate solution of system by name of HAM as:

$$
u(x, t)=u_{0}(x, t)+\sum_{m=1}^{\infty} u_{m}(x, t)
$$

$$
=u_{0}(x, t)+u_{1}(x, t)+u_{2}(x, t)+\cdots,
$$

and

$$
v(x, t)=v_{0}(x, t)+\sum_{m=1}^{\infty} v_{m}(x, t) \quad=v_{0}(x, t)+v_{1}(x, t)+v_{2}(x, t)+\cdots .
$$

Also, the advantages of normal HAM are summarized in three points as in [8].

\section{Application}

when we set $\alpha_{1}(t)=p(t)=-1 / 2, \beta_{1}(t)=\alpha_{2}(t)=\beta_{2}(t)=2$ and $\gamma_{1}(t)=\gamma_{2}(t)=0$, then the system (1) reduces to the Boussinesq-Burgers (B-B) system $[9,10]$.The B-B equations are:

$$
\begin{aligned}
& u_{t}=-2 u u_{x}+1 / 2 v_{x}, \\
& v_{t}=1 / 2 u_{x x x}-2(u v)_{x} .
\end{aligned}
$$

The exact solitary wave solution of system (8) as in [11] are:

$$
\begin{aligned}
& u(x, t)=\frac{c k}{2}+\frac{c k}{2} \tanh \left(\frac{c k^{2} t-k x-\ln (b)}{2}\right), \\
& v(x, t)=\frac{-k^{2}}{8} \operatorname{sech}^{2}\left(\frac{k x-c k^{2} t+\ln (\mathrm{b})}{2}\right),
\end{aligned}
$$

where $c, k$ are arbitrary constants and $0<b<\infty$, with the initial conditions

$$
\begin{aligned}
& u(x, 0)=\frac{c k}{2}+\frac{c k}{2} \tanh \left(\frac{-k x-\ln (b)}{2}\right), \\
& v(x, 0)=\frac{-k^{2}}{8} \operatorname{sech}^{2}\left(\frac{k x+\ln (b)}{2}\right) .
\end{aligned}
$$

In this section, we demonstrate the analysis of numerical methods [MVIM \& HAM] by applying methods to the system of nonlinear partial differential Equations (8)

Note that all the numerical results for $u(x, t)$ and $v(x, t)$ that we calculate it by using MVIM and HAM technique and all Figures and Tables, we used MATHEMATICA software due the simplicity and powerful manipulation.

For numerical result of system (8) and using four terms approximation solution by MVIM are:

$$
\begin{gathered}
u(x, t)=u_{3}(x, t) . \\
=\frac{1}{4}\left(-\tanh \left(\frac{1}{2}(x-\log (2))\right)-1\right)-\frac{1}{16} \operatorname{sech}^{2}\left(\frac{1}{2}(x-\log (2))\right) \cdot t+\frac{1}{8} \sinh ^{4}\left(\frac{1}{2}(x-\log (2))\right) \operatorname{csch}^{3}(x-\log (2)) \cdot t^{2}+ \\
\frac{1}{5160960}\left(26880 \operatorname{sech}^{2}\left(\frac{1}{2}(x-\log (2))\right)-5040 \sinh (2 x-\log (4)) \operatorname{sech}^{6}\left(\frac{1}{2}(x-\log (2))\right)+\right. \\
15120 \sinh (x) \operatorname{sech}^{4}\left(\frac{1}{2}(x-\log (2))\right)-13440 \tanh \left(\frac{1}{2}(x-\log (2))\right) \operatorname{sech}^{4}\left(\frac{1}{2}(x-\log (2))\right)- \\
25200 \cosh (x) \operatorname{sech}^{4}\left(\frac{1}{2}(x-\log (2))\right)+215040 \sinh ^{4}\left(\frac{1}{2}(x-\log (2))\right) \operatorname{csch}^{3}(x-\log (2))-
\end{gathered}
$$


$5040 \sinh (x) \tanh \left(\frac{1}{2}(x-\log (2))\right) \operatorname{sech}^{4}\left(\frac{1}{2}(x-\log (2))\right)+8400 \cosh (x) \tanh \left(\frac{1}{2}(x-\log (2))\right) \operatorname{sech}^{4}\left(\frac{1}{2}(x-\right.$ $\log (2)))) \cdot t^{3}$

And

$$
\begin{gathered}
v(x, t)=v_{3}(x, t) . \\
=-\frac{1}{82575360} \operatorname{sech}^{12}\left(\frac{1}{2}(x-\log (2))\right)(4233600 \cosh (x-\log (2))+201600 \cosh (4(x-\log (2)))+
\end{gathered}
$$
$20160 \cosh (5(x-\log (2)))+2419200 \cosh (2 x-\log (4))+907200 \cosh (3 x-\log (8))+2540160)-$ $\frac{1}{82575360}(-423360 \sinh (x-\log (2))-80640 \sinh (4(x-\log (2)))-10080 \sinh (5(x-\log (2)))-$ $483840 \sinh (2 x-\log (4))-272160 \sinh (3 x-\log (8))) \operatorname{sech}^{12}\left(\frac{1}{2}(x-\log (2))\right) \cdot t-\frac{1}{82575360} \operatorname{sech}^{12}\left(\frac{1}{2}(x-\right.$ $\log (2)))(-317520 \cosh (x-\log (2))+10080 \cosh (4(x-\log (2)))+2520 \cosh (5(x-\log (2)))-$ $120960 \cosh (2 x-\log (4))-7560 \cosh (3 x-\log (8))-211680) \cdot t^{2}-\frac{1}{82575360}(52920 \sinh (x-\log (2))+$ $1680 \sinh (4(x-\log (2)))-420 \sinh (5(x-\log (2)))+50400 \sinh (2 x-\log (4))+18900 \sinh (3 x-$ $\log (8))) \operatorname{sech}^{12}\left(\frac{1}{2}(x-\log (2))\right) \cdot t^{3}$,

For numerical result of system (8) and using four terms approximation solution by HAM are:

$$
\begin{aligned}
& u(x, t, \hbar)=u_{0}(x, t, \hbar)+u_{1}(x, t, \hbar)+u_{2}(x, t, \hbar)+u_{3}(x, t, \hbar) \\
&=\frac{1}{3072}\left(t \hbar \operatorname { s e c h } ^ { 5 } ( \frac { 1 } { 2 } ( x - \operatorname { l o g } ( 2 ) ) ) \left(-6\left(\left(t^{2}+6 t-24\right) \hbar^{2}+6(t-8) \hbar-24\right) \cosh \left(\frac{1}{2}(x-\log (2))\right)\right.\right. \\
&+ 2\left(\left(t^{2}+6 t+24\right) \hbar^{2}+6(t+8) \hbar+24\right) \cosh \left(\frac{3}{2}(x-\log (2))\right) \\
&-3 t \hbar(\hbar+1)\left(-8 \sinh \left(\frac{1}{2}(x-\log (2))\right)-8 \sinh \left(\frac{3}{2}(x-\log (2))\right)-3 \sinh \left(\frac{1}{2}(3 x-\log (2))\right)\right. \\
&+\left.\left.9 \sinh \left(\frac{1}{2}(x+\log (2))\right)+5 \cosh \left(\frac{1}{2}(3 x-\log (2))\right)-15 \cosh \left(\frac{1}{2}(x+\log (2))\right)\right)\right) \\
&+ 192 t \hbar \operatorname{sech}^{2}\left(\frac{1}{2}(x-\log (2))\right) \\
&+ 48 t \hbar \operatorname{sech}^{2}\left(\frac{1}{2}(x-\log (2))\right)\left(t \hbar \tanh \left(\frac{1}{2}(x-\log (2))\right)+4 \hbar+4\right) \\
&-\left.768 \tanh \left(\frac{1}{2}(x-\log (2))\right)-768\right)
\end{aligned}
$$

And

$$
\begin{gathered}
v(x, t, \hbar)=v_{0}(x, t, \hbar)+v_{1}(x, t, \hbar)+v_{2}(x, t, \hbar)+v_{3}(x, t, \hbar) . \\
t \hbar\left(\hbar \left(16 t^{2} \hbar \operatorname{sech}^{6}\left(\frac{1}{2(x-\log (2))}\right)+1024 t^{2} \hbar \sinh ^{10}\left(\frac{1}{2(x-\log (2))}\right) \operatorname{csch}^{6}(x-\log (2))-\right.\right.
\end{gathered}
$$

$5632 t^{2} \hbar \sinh ^{8}\left(\frac{1}{2(x-\log (2))}\right) \operatorname{csch}^{6}(x-\log (2))-24 t \hbar \operatorname{sech}^{4}\left(\frac{1}{2(x-\log (2))}\right)(\cosh (x-\log (2))-2)+$ $3072 t \hbar \sinh ^{8}\left(\frac{1}{2(x-\log (2))}\right) \operatorname{csch}^{5}(x-\log (2))-6144 t \hbar \sinh ^{6}\left(\frac{1}{2(x-\log (2))}\right) \operatorname{csch}^{5}(x-\log (2))+24 \operatorname{sech}^{4}\left(\frac{1}{2(x-\log (2))}\right)(2(\hbar+$ 1) $(3 \cosh (x)-5 \sinh (x))-t \hbar(\cosh (x-\log (2))-2))+t\left(\frac{512 e^{2 x}\left(t\left(e^{x}-2\right)^{2} \hbar+6\left(e^{2 x}-4\right)(\hbar+1)\right)}{\left(e^{x}+2\right)^{6}}+\frac{256 e^{2 x}\left(e^{2 x}((t+6) \hbar+6)-4(t-6) \hbar+24\right)}{\left(e^{x}+2\right)^{5}}-\right.$ $20 \operatorname{thsech}^{6}\left(\frac{1}{2(x-\log (2))}\right)-16 \operatorname{thsech}^{4}\left(\frac{1}{2(x-\log (2))}\right)+8 \tanh \left(\frac{1}{2(x-\log (2))}\right) \operatorname{sech}^{4}\left(\frac{1}{2(x-\log (2))}\right)\left(\operatorname{th} \tanh \left(\frac{1}{2(x-\log (2))}\right)+6 \hbar+6\right)+$

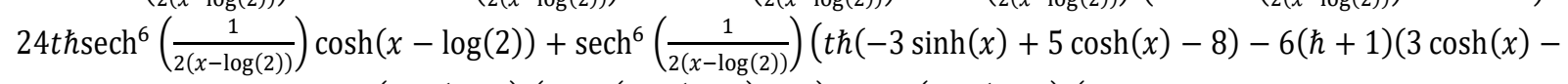
$5 \sinh (x)))-4 \tanh \left(\frac{1}{2(x-\log (2))}\right)\left(\tanh \left(\frac{1}{2(x-\log (2))}\right)+1\right) \operatorname{sech}^{4}\left(\frac{1}{2(x-\log (2))}\right)((-3 t \hbar+30 \hbar+30) \sinh (x)+$ $((5 t-18) \hbar-18) \cosh (x)-8 t \hbar))+3072 t \sinh ^{8}\left(\frac{1}{2(x-\log (2))}\right) \operatorname{csch}^{5}(x-\log (2))-6144 t \sinh ^{6}\left(\frac{1}{2(x-\log (2))}\right) \operatorname{csch}^{5}(x-$ 


$$
\begin{gathered}
\left.\log (2)))-48 \operatorname{sech}^{4}\left(\frac{1}{2(x-\log (2))}\right)(t \hbar \cosh (x-\log (2))-2 t \hbar+5 \hbar \sinh (x)-3(\hbar+1) \cosh (x)+5 \sinh (x))\right)- \\
3072 t \hbar \sinh ^{4}\left(\frac{1}{2(x-\log (2))}\right) \operatorname{csch}^{3}(x-\log (2))-48 t \hbar \operatorname{sech}^{4}\left(\frac{1}{2(x-\log (2))}\right)(t \hbar \cosh (x-\log (2))-2 t \hbar+5 \hbar \sinh (x)- \\
3(\hbar+1) \cosh (x)+5 \sinh (x))-768 \operatorname{sech}^{2}\left(\frac{1}{2(\log (2)-x)}\right) .
\end{gathered}
$$

\subsection{Numerical Results (Tables, Figures)}

Table 1 and 2 shows the absolute error between the exact solution and approximate solutions by MVIM, HAM

\begin{tabular}{|c|c|c|}
\hline $\mathbf{u}(x, t)$ & | Exact - MVIM | & $\begin{array}{c}\mid \text { Exact }- \text { HAM } \mid \\
\hbar=-1.015\end{array}$ \\
\hline$(0.4,0.0)$ & $5.55112 \times 10^{-17}$ & 0 \\
\hline$(0.4,0.2)$ & $1.34874 \times 10^{-7}$ & $3.20675 \times 10^{-7}$ \\
\hline$(0.4,0.4)$ & $2.01176 \times 10^{-6}$ & $1.63761 \times 10^{-6}$ \\
\hline$(0.4,0.6)$ & $9.42085 \times 10^{-6}$ & $2.77748 \times 10^{-6}$ \\
\hline$(0.4,0.8)$ & $2.73014 \times 10^{-5}$ & $1.41787 \times 10^{-6}$ \\
\hline$(0.4,1.0)$ & $6.05078 \times 10^{-5}$ & $4.67858 \times 10^{-6}$ \\
\hline Least square error & $4.49938 \times 10^{-9}$ & $3.43985 \times 10^{-11}$ \\
\hline
\end{tabular}
for $u(x, t)$ and $v(x, t)$ respectively, when $x=0.4$ (Arbitrary chosen) and $t \in[0,1]$.

Table (1)

\begin{tabular}{|c|c|c|}
\hline $\mathbf{v}(x, t)$ & | Exact - MVIM | & $\begin{array}{c}\mid \text { Exact - HAM | } \\
\hbar=-0.927\end{array}$ \\
\hline$(0.4,0.0)$ & $2.77556 " \times 10^{-17}$ & 0 \\
\hline$(0.4,0.2)$ & $4.41678 \times 10^{-7}$ & $4.3911 \times 10^{-6}$ \\
\hline$(0.4,0.4)$ & $7.22664 \times 10^{-6}$ & $1.81655 \times 10^{-5}$ \\
\hline$(0.4,0.6)$ & $3.72929 \times 10^{-5}$ & $3.13989 \times 10^{-5}$ \\
\hline$(0.4,0.8)$ & $1.19764 \times 10^{-4}$ & $2.19814 \times 10^{-5}$ \\
\hline$(0.4,1.0)$ & $2.96173 \times 10^{-4}$ & $4.46077 \times 10^{-5}$ \\
\hline Least square error & $1.03505 \times 10^{-7}$ & $3.80819 \times 10^{-9}$ \\
\hline
\end{tabular}

\section{Table (2)}

Also, the Fig. 1, Fig. 2 And Fig. 3 Below are the surfaces for the exact solution of B.B system, MVIM and HAM respectively, when $x \in[-10,10]$ and $t \in[0,1]$.

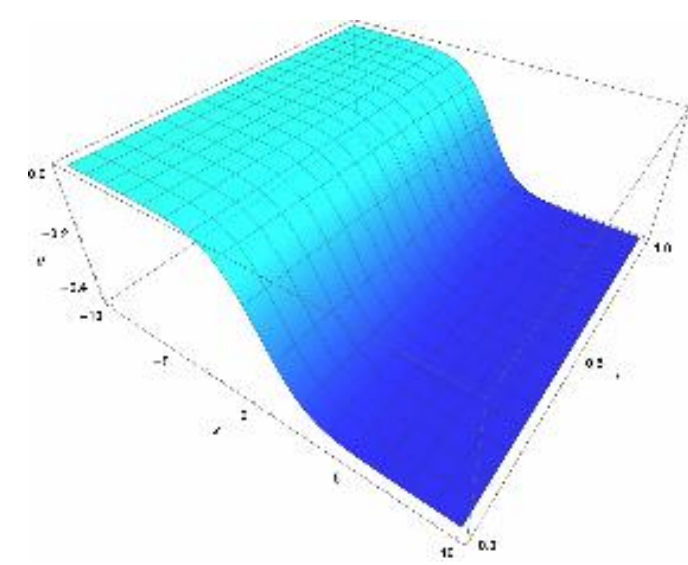

(a) $u(x, t)$

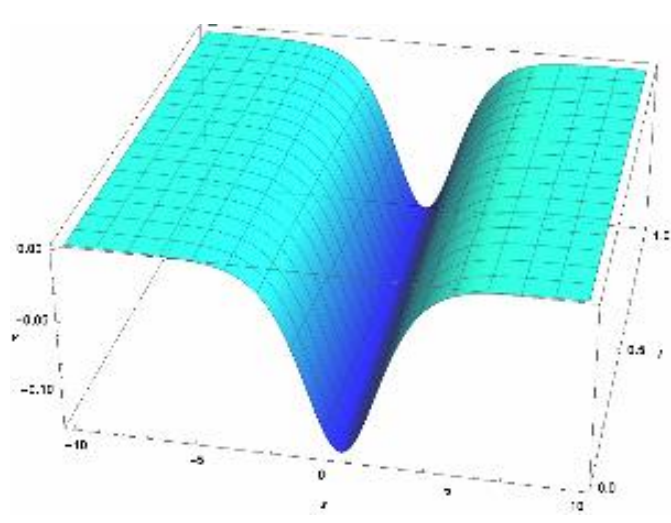

(b) $v(x, t)$

Fig (1): The surfaces of exact solutions 


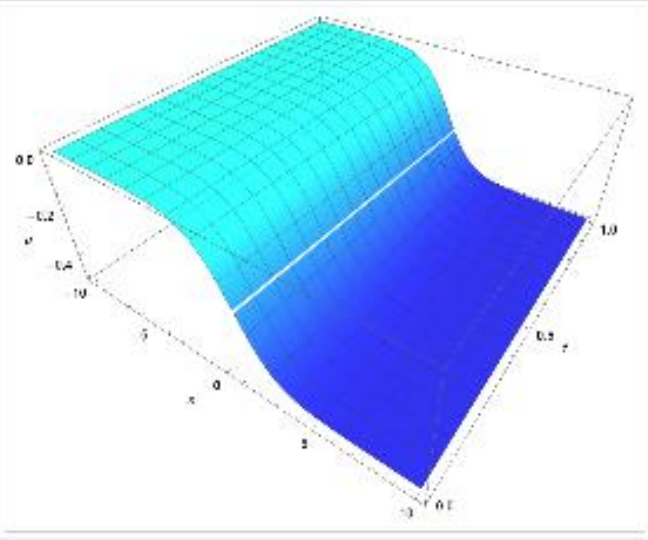

(a) $u(x, t)$

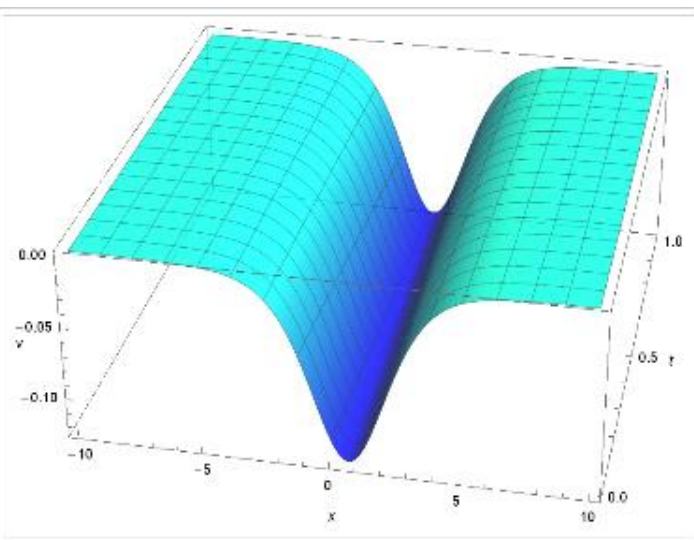

(b) $v(x, t)$

Fig (2): The surfaces of MVIM solutions

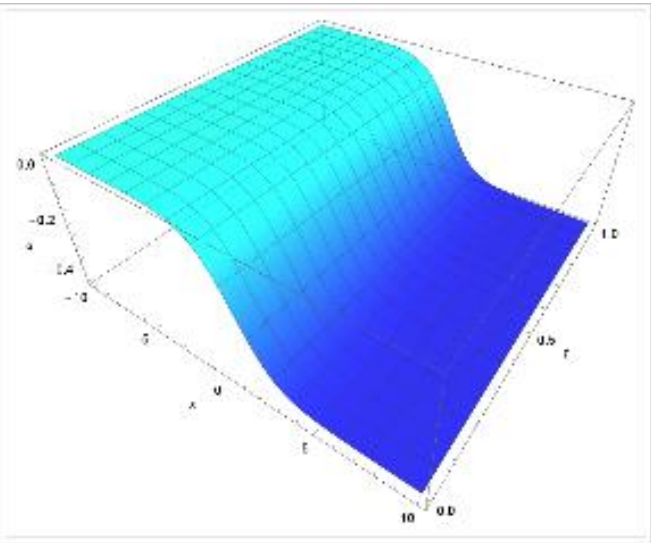

(a) $u(x, t)$

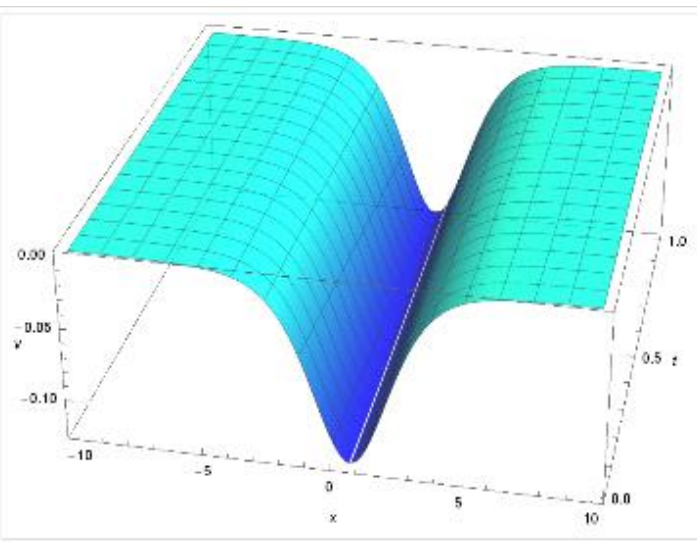

(b) $v(x, t)$

Fig (3): The surfaces of HAM solutions

The Curves in Fig. 4 and Fig. 5 shows us how the MVIM and HAM curves is close to the exact solution curve, when $x=\mathbf{0 . 4}$ and $t \in[0,1]$.

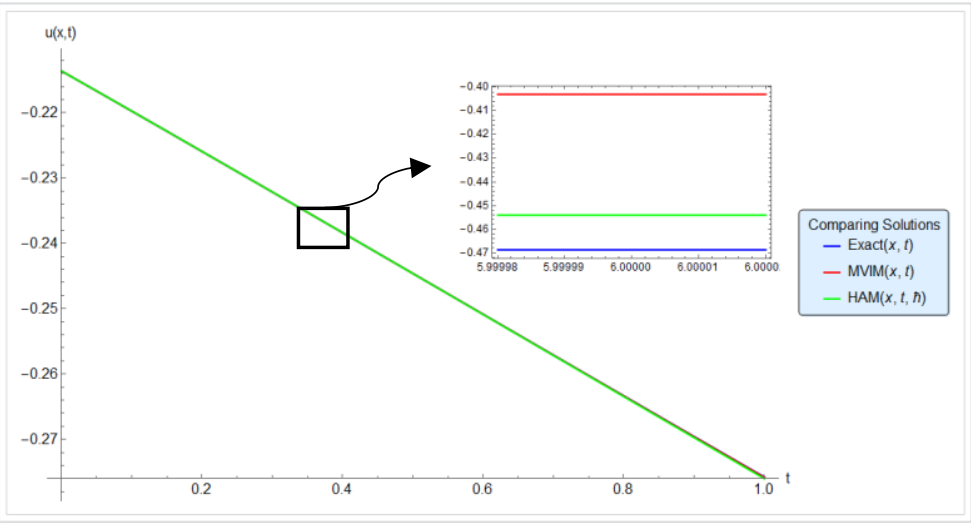

Fig (4): The curves of $u(x, t)$ 


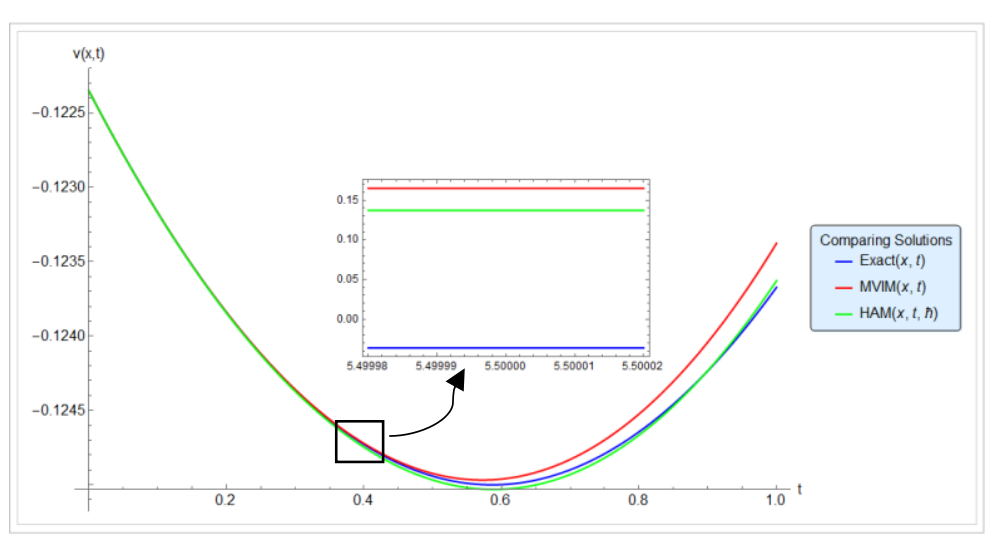

Fig (5): The curves of $v(x, t)$

\section{Conclusion}

In this paper the Variable-Coefficient Variant Boussinesq system was solved numerically by using Modified Variational Iteration Method, Homotopy Analysis Method, as an example we took the (B-B) equation and Comparison between our solutions and the exact solution shows that both the methods are effective and accurate in solving (B-B) equation, whereas HAM is accurate in compared to MVIM for $u(x, t)$ and $v(x, t)$ directions. HAM is the best and fastest than MVIM because it does not require a large amounts of computer memory or time, and it contains a certain auxiliary parameter $\hbar$ which provides us a simple way to control the convergence region and rate of convergence of the series solution as shown in Tables $\{1,2\}$ and the Figures $\{5,6\}$. Moreover, MVIM has the same results as Adomian Decomposition Method (ADM) as in [12] this conclusion satisfied to solve this type of System of Non-Linear Partial Differential Equations.

\section{References:}

[1] T. A. Abassy, M. A. El-Tawil \& H. El Zoheiry, Toward a Modified Variational Iteration Method, Journal of Computational and Applied Mathematics, 207(1)(2007), 137-147, https://doi.org/10.1016/j.cam.2006.07.019

[2] F. H. Easif, Adomain Decomposition Method for Solving Non Linear Partial Differential Equations, IOSR Journal of Mathematics, 10(5)(2014), 60-66, https://doi.org/10.9790/5728-10556066

[3] A. K. Gupta \& Ray, S. Saha, Comparison between Homotopy Perturbation Method and Optimal Homotopy Asymptotic Method for the Soliton Solutions of Boussinesq-Burger Equations, Computers \& Fluids, 103(2014), 34-41, https://doi.org/10.1016/j.compfluid.2014.07.008

[4] S. Liao, Homotopy Analysis Method in Nonlinear Differential Equations, Springer Berlin Heidelberg, (2012).

[5] B. Mahmood, A. Manaa Saad \& Fadhil H. Easif, Homotopy Analysis Method for Solving Nonlinear Diffusion Equation with Convection Term, International Journal of Applied Mathematical Research, 3(3)(2014)244-250, https://doi.org/10.14419/ijamr.v3i3.2899

[6] J. Mei \& Z. Ma, N-Fold Darboux Transformation and Multi-Soliton Solutions for the Classical BoussinesqBurgers System, Applied Mathematics and Computation, 219 (11) (2013), 6163-6169, https://doi.org/10.1016/j.amc.2012.12.044

[7] M. D. Mikhailov \& M. N. Ozisik, Unified Analysis and Solutions of Heat and Mass Diffusion, Dover Publications, (1984).

[8] Ahmed J. Sabali, Saad A. Manaa and Fadhil H. Easif, Adomian and Adomian-Padé Technique for Solving Variable Coefficient Variant Boussinesq System, Science Journal of University of Zakho, 6(3)(2018), 108-112, https://doi.org/10.25271/sjuoz.2018.6.3.514

[9] K. Singh \& R. K. Gupta, Exact Solutions of a Variant Boussinesq System, International Journal of Engineering Science, 44(18-19)(2006), 1256-1268, https://doi.org/10.1016/j.ijengsci.2006.07.009

[10] E. T. Swartz \& R. O.Pohl, Thermal Resistance at Interfaces, Applied Physics Letters, 51(26)(1987), 2200-2202.

[11] Y.-H. Wang, CTE Method to the Interaction Solutions of Boussinesq-Burgers Equations, Applied Mathematics Letters, 38 (2014), 100-105, https://doi.org/10.1016/j.aml.2014.07.014

[12] Xindong. Zhang \& Ma. Changxiu, On exact solution of Poisson equation with boundary conditions by the homotopy analysis method, Computer Application and System Modeling (ICCASM), 6 (2010), https://doi.org/10.1109/iccasm.2010.5620203 